\title{
ImmunoHorizons
}

\section{Role of Mitochondrial Dynamics in Microglial Activation and Metabolic Switch}

Alejandro Montilla, Asier Ruiz, Mar Marquez, Amanda Sierra, Carlos Matute and Maria Domercq

ImmunoHorizons 2021, 5 (8) 615-626

doi: https://doi.org/10.4049/immunohorizons.2100068

http://www.immunohorizons.org/content/5/8/615

This information is current as of October 8, 2021.

\section{Supplementary http://www.immunohorizons.org/content/suppl/2021/08/10/immunohorizon \\ Material s.2100068.DCSupplemental}

References This article cites $\mathbf{5 2}$ articles, 8 of which you can access for free at: http://www.immunohorizons.org/content/5/8/615.full\#ref-list-1

Email Alerts Receive free email-alerts when new articles cite this article. Sign up at: http://www.immunohorizons.org/alerts

ImmunoHorizons is an open access journal published by

The American Association of Immunologists, Inc.,

1451 Rockville Pike, Suite 650, Rockville, MD 20852

All rights reserved.

ISSN 2573-7732.

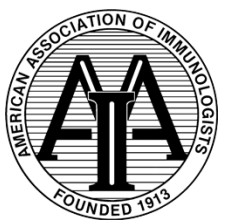




\title{
Role of Mitochondrial Dynamics in Microglial Activation and Metabolic Switch
}

\author{
Alejandro Montilla, ${ }^{*, \dagger}$ Asier Ruiz, ${ }^{*, \dagger}$ Mar Marquez, ${ }^{*}$ Amanda Sierra, ${ }^{*, \neq}$ Carlos Matute, ${ }^{,, \dagger}$ and Maria Domercq ${ }^{*, \dagger}$ \\ ${ }^{*}$ Achucarro Basque Center for Neuroscience and Department of Neuroscience, University of the Basque Country, Leioa, Spain; ${ }^{\dagger}$ Centro de Investigación \\ Biomédica en Red de Enfermedades Neurodegenerativas, Leioa, Spain; and ${ }^{\ddagger} \mid$ kerbasque Foundation, Bilbao, Spain
}

\section{ABSTRACT}

Microglia act as sensors of injury in the brain, favoring its homeostasis. Their activation and polarization toward a proinflammatory phenotype are associated with injury and disease. These processes are linked to a metabolic reprogramming of the cells, characterized by high rates of glycolysis and suppressed oxidative phosphorylation. This metabolic switch can be reproduced in vitro by microglial stimulation with LPS plus IFN- $\gamma$. To understand the mechanisms regulating mitochondrial respiration abolishment, we examined potential alterations in mitochondrial features during this switch using rat primary microglia. Cells did not show any change in mitochondrial membrane potential, suggesting a limited impact in the mitochondrial viability. We provide evidence that reverse operation of $\mathrm{F}_{0} \mathrm{~F}_{1}$-ATP synthase contributes to mitochondrial membrane potential. In addition, we studied the possible implication of mitochondrial dynamics in the metabolic switch using the mitochondrial division inhibitor-1 (Mdivi-1), which blocks dynamin-related protein 1 (Drp1)-dependent mitochondrial fission. Mdivi-1 significantly reduced the expression of proinflammatory markers in LPS plus IFN- $\gamma$-treated microglia. However, this inhibition did not lead to a recovery of the oxidative phosphorylation ablation by LPS plus IFN$\gamma$ or to a microglia repolarization. Altogether, these results suggest that Drp1-dependent mitochondrial fission, although potentially involved in microglial activation, does not play an essential role in metabolic reprogramming and repolarization of microglia.

ImmunoHorizons, 2021, 5: 615-626.

\section{INTRODUCTION}

Microglial cells are the resident immune cells of the CNS. They contribute to the tissue development, integrity and homeostasis through an active surveillance process $(1,2)$ that can lead to different states of activation. Microglia display an enormous plasticity of responses to injury, ranging from effects that may contribute to neuroinflammation and, eventually, tissue damage, to essential responses for regenerative processes (3). These mechanisms are associated with a broad spectrum of activation states, which are in turn related to molecular changes and adaptations (4). One of these adaptive mechanisms is the metabolic reprogramming of microglia and other immune cells in response to immune activation (5-8). Similar to macrophages $(6,7)$, microglia exposed to proinflammatory stimulus shift their metabolism from oxidative phosphorylation (OXPHOS) to aerobic glycolysis $(9,10)$, in an event similar to the Warburg effect suffered by cancer cells (7). This metabolic reprogramming

Received for publication July 19, 2021. Accepted for publication July 20, 2021.

Address correspondence and reprint requests to: Dr. Maria Domercq, Departamento de Neurociencias, Universidad del País Vasco, Barrio Sarriena s/n, E-48940 Leioa, Spain. E-mail address: maria.domercq@ehu.es

ORCID: 0000-0002-4918-9276 (M.D.).

M.D. contributed to the conception and design of the study, data interpretation, and manuscript writing. A.M. contributed to the data acquisition and analysis, data interpretation, and manuscript writing. A.R. assisted with the data acquisition and interpretation. M.M. and A.S. contributed the analysis of mitochondrial morphology. C.M. substantially contributed to the revision of the work. All authors contributed to the manuscript revision, read, and approved the submitted version.

Abbreviations used in this article: ANT, adenine nucleotide translocase; Arg1, arginase 1; Drp-1, dynamin-related protein 1; ECAR, extracellular acidification rate;

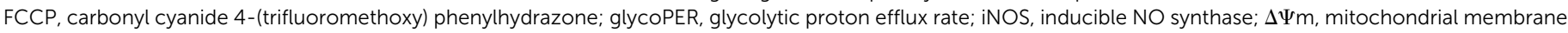
potential; Mdivi-1, mitochondrial division inhibitor 1; MNR, mannose receptor; OCR, oxygen consumption rate; OXPHOS, oxidative phosphorylation; qPCR, quantitative PCR; Rh123, rhodamine 123 dye; ROS, reactive oxygen species.

The online version of this article contains supplemental material.

This article is distributed under the terms of the CC BY-NC-ND 4.0 Unported license.

Copyright () 2021 The Authors 
is essential to activate cellular defense mechanisms and to manage various microenvironments in inflamed tissue (7, 11-13). Moreover, these cells overexpress inflammatory markers, such as IL- $1 \beta$, TNF- $\alpha$, or inducible NO synthase (iNOS), which takes part in the metabolism of arginine. In contrast, macrophages exposed to anti-inflammatory stimuli are primarily characterized by OXPHOS and increased fatty acid oxidation for ATP synthesis $(6,12)$, a fact that has not been corroborated in microglia cells. This anti-inflammatory activation is commonly accompanied by an increase in the expression of specific markers, such as arginase 1 (Arg1), which competes with iNOS to hydrolyze arginine to ornithine (14), or the mannose receptor (MNR).

As mitochondria are crucial organelles in OXPHOS-related metabolism of the cells, we hypothesized that disbalances in their integrity or dynamics may contribute to microglia metabolic reprogramming toward a glycolytic phenotype. Mitochondrial dynamics, involving processes of both fusion and fission, are known to contribute to cell homeostasis and the maintenance of other mitochondrial functions (15). Basal mitochondrial fission is required for mitochondrial trafficking to synapses, mitochondrial quality control, and brain development (16). Misbalances in these dynamic mechanisms are thought to contribute to the development of various neurologic disorders (17-19). Moreover, these processes are associated with the production of reactive oxygen species (ROS). Mitochondrial fission is triggered by the dynamin-related protein 1 (Drpl) recruitment from the cytosol to the outer mitochondrial membrane, which is mediated by different adaptor proteins (20, 21). Lately, the pharmacological inhibition of Drpl with the mitochondrial division inhibitor 1 (Mdivi-1), a quinazolinone derivative, has become a promising strategy to analyze the physiological and pathological role of mitochondrial fission (22).

We tested whether mitochondria are functionally affected during metabolic reprogramming conditions. Moreover, we assessed whether mitochondrial fission is involved in microglia metabolic reprogramming. We used Mdivi-1 to block Drp1dependent mitochondrial fission in vitro and found that, whereas this inhibition leads to a modulation of the expression of inflammatory markers in microglial cells, this is not sufficient to alter the metabolic switch suffered by the cells, in sharp contrast with the results reported in a previous study (9). Furthermore, as Mdivi-1 treatment generated a decrease in iNOS expression, we have assessed whether this outcome was sufficient to favor a repolarization process in microglia.

\section{MATERIALS AND METHODS}

\section{Animals}

All experiments were performed according to the procedures approved by the Ethics Committee of the University of the Basque Country. Animals were handled in accordance with the European Communities Council Directive and were kept under conventional housing conditions $\left(22 \pm 2{ }^{\circ} \mathrm{C}, 55 \pm 10 \%\right.$ humidity, and 12-h day/night cycle) at the animal facilities. The study was carried out in compliance with the Animal Research: Reporting of In Vivo Experiments guidelines. All possible efforts were made to minimize animal suffering and the number of animals used.

\section{Microglial culture}

Primary mixed glial cultures were prepared from the cerebral cortex of neonatal rats (P0-P2). After 10-15 d in culture, microglia were isolated by mechanical shaking (400 rpm, $1 \mathrm{~h}$ ) and purified by plating them on noncoated bacterial grade Petri dishes (Sterilin; Thermo Fisher Scientific), as previously described (23). Microglial cells obtained with this procedure were cultured in DMEM (Life Technologies) supplemented with 10\% FBS (Life Technologies). Cultures of microglia were practically pure of microglial cells (>99\%) (24).

Microglial cells were polarized with specific stimuli. To generate an anti-inflammatory phenotype in these cells, they were treated for $24 \mathrm{~h}$ with IL-4 (20 ng/ml; PeproTech) and IL13 (50 ng/ml; PeproTech). In contrast, proinflammatory microglia were generated with a treatment of both LPS $(10 \mathrm{ng} / \mathrm{ml}$; Sigma-Aldrich) and IFN- $\gamma$ (20 ng/ml; PeproTech) for $24 \mathrm{~h}$. In the experiments for the study of the proinflammatory activation time course, the LPS plus IFN- $\gamma$ treatment was maintained for 2,6 , or $24 \mathrm{~h}$ prior to the metabolic analysis. The repolarization protocol implied a $24-\mathrm{h}$ proinflammatory activation followed by a treatment with the anti-inflammatory stimuli for the same amount of time. Mdivi-1 (50 $\mu \mathrm{M}, 24 \mathrm{~h}$ ) was applied alone or in combination with pro- and anti-inflammatory factors.

\section{Immunofluorescence analysis}

Several immunocytochemistry assays were performed on the cells in different conditions. Cells in culture were fixed in $4 \%$ p-formaldehyde in PBS and processed for immunocytochemistry, as previously described (25). Primary Abs were used were follows: Ibal (1:500; Wako Chemicals), iNOS (1:500; BD Biosciences), a proinflammatory marker, and MNR C type 1 (MRC1; 1:1000; Abcam), specific for anti-inflammatory cells. As secondary Abs, we used goat anti-rabbit Alexa Fluor 488 (1:250; Invitrogen) and goat anti-mouse Alexa Fluor 594 (1:250; Invitrogen). The morphology of microglial cells was analyzed using the Ibal immunofluorescence, as the whole soma was stained by it. The circularity of the cell was calculated as defined in the ImageJ software; a circular shape would approach a value of 1 . All the image analysis was performed with the ImageJ software (National Institutes of Health). Immunoreactivity of the different markers was calculated as the fluorescence intensity normalized to the number of cells in the selected field of view.

\section{Cell viability assays}

Microglial viability was assessed using both the calcein AM dye (Invitrogen) and the CytoTox 96 Non-Radioactive Cytotoxicity Assay (Promega).

For the performance of the calcein assay, cells were incubated with $0.5 \mu \mathrm{M}$ of the dye for $30 \mathrm{~min}$ at $37^{\circ} \mathrm{C}$. The wells 
were then washed with PBS, and the number of viable cells were calculated with a Synergy HT fluorimeter/spectrophotometer reader (BIO-TEK INSTRUMENTS) with 485-nm excitation and at $528 \mathrm{~nm}$ of emission wavelengths. A well with PBS was also measured in each experiment to subtract its values as a background control. The results are expressed as the relative percentage of cellular death with respect to nontreated microglial cells.

For the CytoTox 96 Non-Radioactive Cytotoxicity Assay, culture supernatants were harvested from the plate, mixed with the specific assay buffer and incubated for $30 \mathrm{~min}$ at $37^{\circ} \mathrm{C}$. The reaction was then stopped, and the absorbance at $490 \mathrm{~nm}$ was immediately recorded in the fluorimeter. The levels of absorbance are associated with the amount of lactate dehydrogenase in the medium, a stable cytosolic enzyme that is released upon cellular death and its consequent lysis. All the data obtained through both assays was analyzed using Gen5 software (BIO-TEK INSTRUMENTS).

\section{ROS analysis}

The production of ROS by control microglia and microglia treated with LPS and IFN- $\gamma$ was measured using the CM$\mathrm{H}_{2}$ DCFDA dye (Invitrogen). Specifically, cells were incubated with the dye for $30 \mathrm{~min}$. The wells were then washed with PBS, and the number of viable cells were calculated with a Synergy HT fluorimeter/spectrophotometer reader (BIO-TEK INSTRUMENTS) with $485-\mathrm{nm}$ excitation and at $528 \mathrm{~nm}$ of emission wavelengths, following the guidelines of the manufacturer.

\section{Quantitative RT-PCR}

Total RNA of microglial cells was extracted using TRIzol (Invitrogen) according to the manufacturer's instructions. Afterwards, $1 \mu \mathrm{g}$ of this RNA was used to perform a retrotranscription protocol, using SuperScript III Reverse Transcriptase (200 U/ $\mu \mathrm{l}$; Invitrogen) and random hexamers as primers (Promega).

Real-time quantitative PCRs (qPCRs) were conducted in a Bio-Rad Laboratories CFX96 real-time PCR detection system, as previously described (26). The reactions were performed using SYBR Green as a DNA-binding dye and specific primers for anti-inflammatory and proinflammatory markers (Table I). These primers were designed using Primer Express Software (Applied Biosystems) at exon junctions to avoid genomic DNA amplification. The cycling conditions comprised 3 min of polymerase activation at $95^{\circ} \mathrm{C}$ and 40 cycles consisting of $10 \mathrm{~s}$ at $95^{\circ} \mathrm{C}$ and $30 \mathrm{~s}$ at $60^{\circ} \mathrm{C}$. After every qPCR reaction, the melting curve was assessed to check the specificity of the amplification. The amount of cDNA was quantified using a standard curve from a pool of cDNA obtained from the different conditions of each experiment. Subsequently, the results were normalized using a normalization factor based on the geometric mean of housekeeping genes (Table I) obtained for each condition using the geNorm v3.5 software (27).

\section{Mitochondrial membrane potential and mitochondrial calcium measurements}

For quantification of mitochondrial membrane potential $(\Delta \Psi \mathrm{m})$, we use rhodamine 123 dye (Rh123) under quenching concentrations, a reliable dye for confocal microscopy analysis of $\Delta \Psi \mathrm{m}$ (28-30). Microglial cells were loaded with Rh123 (10 $\mu \mathrm{M})$ for $15 \mathrm{~min}$ in medium without phenol red. After this, microglia were washed for $10 \mathrm{~min}$ in the same medium. Living cell imaging was performed with a $\times 63$ objective in an inverted Leica TCS SP2-AOBS confocal microscope at an acquisition rate of one frame every $15 \mathrm{~s}$ for $5 \mathrm{~min}$. In this short-term protocol, no cell toxicity was detected with Rh123. After obtaining some basal images, carbonyl cyanide 4-(trifluoromethoxy) phenylhydrazone (FCCP) $(1 \mu \mathrm{M})$, an uncoupler of the mitochondrial OXPHOS, was added to the plates, and the increase in the Rh123 fluorescence level was measured to determine the membrane potential. For each plate, a homogenous population of $\sim 20$ cells was selected in the field of view, and the background fluorescence signal was subtracted from the individual values.

For the measurement of the mitochondrial calcium content, time-lapse images were acquired using a $\times 63$ objective in a Leica TCS STED CW SP8 confocal microscope, at an acquisition rate of one frame every $15 \mathrm{~s}$ for $5 \mathrm{~min}$. Cells were loaded with $1 \mu \mathrm{M}$ Fluo-4 (Thermo Fisher Scientific) in HBSS $\left(\mathrm{Ca}^{2+}\right.$ and $\mathrm{Mg}^{2+}$-free) with $0.5 \mu \mathrm{M}$ EGTA for $30 \mathrm{~min}$ at $37^{\circ} \mathrm{C}$. Subsequently, microglial cells were washed, and after obtaining some basal images, $1 \mu \mathrm{M}$ FCCP was added to depolarize the mitochondrial membrane and force the release of $\mathrm{Ca}^{2+}$ from mitochondrial matrix to the cytosol. The analysis was made as described above.

\section{Mitochondrial morphology analysis}

For the quantification of mitochondrial length, living cell imaging was performed on microglial cells loaded with Rh123 (50 $\mathrm{nM}$ ) for $20 \mathrm{~min}$. Mitochondrial images were taken using a Zeiss LSM 800 confocal microscope at $\times 40$ magnification and a pixel resolution of $1024 \times 1024$ and analyzed using the Object Analyzer tool of Huygens image analysis software (version 20.10; Scientific Volume Imaging, B.V.). First, background noise was removed using the maximum fluorescence intensity value of Rh123 in the nuclear region. Afterwards, a size filter was applied to remove objects smaller than mitochondria (38 voxels $\left.=0.06 \mu^{3}\right)(31)$. Next, a watershed segmentation was performed using a $\sigma$ value of 0.2 to separate objects that were not discriminated in the raw images because of the inherent resolution limit of confocal microscopy. After this preprocessing, the average length of each mitochondrial object per cell was calculated.

\section{OXPHOS and glycolysis analysis}

Real-time measurements of oxygen consumption rate (OCR), extracellular acidification rate (ECAR), and glycolytic proton efflux rate (glycoPER) were performed using a Seahorse XFe96 Extracellular Flux Analyzer (Agilent Technologies), following 
manufacturer's instructions to carry out the XF Cell Mito Stress Test Kit or the XF Glycolytic Rate Assay Kit (Agilent Technologies). Microglia cells were seeded as a monolayer in the XF96 microplate. Before the assay, cells were washed and equilibrated in the XF Assay modified DMEM For $30 \mathrm{~min}$ at $37^{\circ} \mathrm{C}$.

For the performance of the XF Cell Mito Stress Test Kit, the real levels of OCR were determined in response to the sequential addition of oligomycin $(2 \mu \mathrm{M})$, FCCP $(1 \mu \mathrm{M})$, and rotenone/antimycin A $(0.5 \mu \mathrm{M})$. Specifically, basal mitochondrial respiration was calculated subtracting the OCR rate after rotenone/antimycin A addition from basal OCR. The spare respiratory capacity was obtained by subtracting the basal respiration level from the maximum rate measurement after addition of FCCP; ATP-linked OCR was determined by subtracting oligomycin-induced OCR from basal OCR. For the acute treatment with LPS plus IFN- $\gamma$, these factors were added to the cells after the basal measurement of OCR and prior to the addition of oligomycin.

For the measurement of the basal glycoPER, the XF Glycolytic Rate Assay Kit was carried out. This test includes the sequential addition of rotenone/antimycin $\mathrm{A}(0.5 \mu \mathrm{M})$, and 2deoxyglucose $(50 \mathrm{mM})$. The glycoPER corresponds to the proton efflux rate derived from glycolysis (discounting the effect of $\mathrm{CO}_{2}$-dependent acidification included in the ECAR measurement) All these parameters were obtained using the specific Agilent Report Generator. For each single experiment, eight replicates were performed.

\section{Data analysis}

All the data shown in the figures are presented as the mean \pm SEM. All the statistical analyses were performed with GraphPad Prism 8.0 (GraphPad Software). Specifically, every comparison between two groups was analyzed using a paired Student two-tailed $t$ test. Comparisons among multiple groups were analyzed by one-way ANOVA followed by Bonferroni multiple comparison tests for post hoc analysis. Statistical significance was considered at $p<0.05$.

\section{RESULTS}

\section{Metabolic reprogramming in microglia is not associated with mitochondrial damage}

To test whether microglia metabolic reprogramming from OXPHOS to glycolysis upon proinflammatory stimulation is associated with mitochondrial damage, we set up an in vitro protocol to study the metabolic switch, and we measured OCR and ECAR in real time as indicative of mitochondrial respiration and glycolysis, respectively. Cells treated with classical proinflammatory stimuli (LPS and IFN- $\gamma$ ) showed a flat profile regarding OXPHOS, and all the parameters (basal OCR, ATPlinked respiration, and spare respiratory capacity) related to this molecular process were practically abolished (Fig. 1A), as previously described (32). This suggests a metabolism shift from OXPHOS to glycolysis to rapidly obtain energy.
Conversely, microglia treated with anti-inflammatory factors (IL-4 and IL-13) showed a significant increase in basal OCR, spare respiratory capacity, and ATP-linked respiration, thus indicating that mitochondrial OXPHOS was boosted (Fig. 1A). In contrast, ECAR measurement showed an increase in basal glycolysis in both proinflammatory and anti-inflammatory microglia (Fig. 1B), suggesting that activated microglia have higher energetic demands. Moreover, inhibition of mitochondrial $\mathrm{F}_{0} \mathrm{~F}_{1}-\mathrm{ATP}$ synthase with oligomycin, thus blocking OXPHOS, increased the glycolytic rate in control and antiinflammatory microglia but not in proinflammatory microglia (Fig. 1B). Finally, the basal ratio between mitochondrial OCR and the glycoPER was significantly diminished in proinflammatory microglia (Fig. 1C). These results further support the idea that proinflammatory microglia metabolism relies exclusively on glycolysis.

In other immune cells, such as dendritic cells, the metabolic switch to glycolysis occurs within minutes after TLR activation with LPS (33). However, microglia acute treatment with LPS did not induce any change in OCR-associated parameters (Fig. 1D). Time course analysis demonstrated that the metabolic switch began to occur at $2 \mathrm{~h}$, was clear at $6 \mathrm{~h}$, and completed at $24 \mathrm{~h}$ (Fig. 1E).

Because OXPHOS takes place in the mitochondrial inner membrane and is determinant for $\mathrm{H}^{+}$movement and $\Delta \Psi \mathrm{m}$ maintenance, the arrest of mitochondrial OXPHOS by proinflammatory stimuli could induce a collapse in $\Delta \Psi \mathrm{m}$. To check whether $\Delta \Psi \mathrm{m}$ was altered in microglia treated with proinflammatory stimuli, we performed live cell imaging of Rh123 fluorescent dye in microglia under "quenching" conditions (28). Addition of the mitochondrial OXPHOS uncoupler FCCP provoked the release of Rh123 from the mitochondria and consequently increased its cytoplasmic fluorescence 2 -fold over baseline (100\%) in control microglia as well as in microglia treated with LPS plus IFN- $\gamma$ (Fig. 2A), indicating that basal mitochondrial potential is maintained in proinflammatory microglia, despite the arrest of OXPHOS. To further assess mitochondrial integrity, we measured resting $\left[\mathrm{Ca}^{2+}\right]_{\text {mit }}$ by recording $\left[\mathrm{Ca}^{2+}\right]_{\text {cyt }}$ upon addition of FCCP in the absence of extracellular $\mathrm{Ca}^{2+}$, which is indicative for $\left[\mathrm{Ca}^{2+}\right]_{\text {mit }}$ (34). Again, we observed no significant differences in the release of mitochondrial calcium to the cytosol after the addition of FCCP (Fig. 2B). Moreover, proinflammatory stimulation of microglia induced a significant increase in ROS production, as revealed with the dichlorofluorescein diacetate (DCFDA; Invitrogen) dye (Fig. 2C), despite the blockage of OXPHOS. All these results suggest that proinflammatory activation and its subsequent lack of OXPHOS activity in microglia are not related to and it does induce any mitochondrial dysfunction or alteration in $\Delta \Psi \mathrm{m}$.

Maintaining $\Delta \Psi \mathrm{m}$ is essential to prevent the release of proapoptotic factors into the cytosol and subsequent cell death. Indeed, despite the blockage of OXPHOS, LPS plus IFN- $\gamma$ stimulation did not induce any significant microglia cell death (Fig. 2D). We hypothesized that $\Delta \Psi \mathrm{m}$ could be maintained in cells 
A

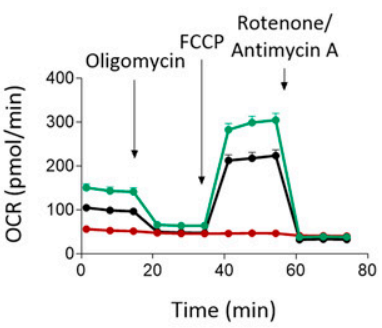

B

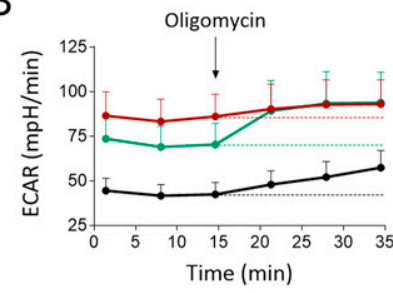

D

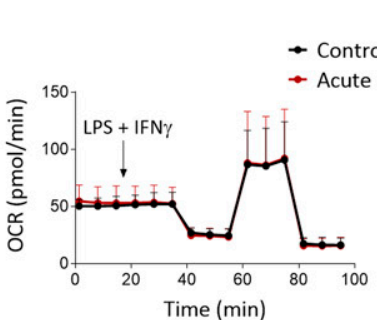

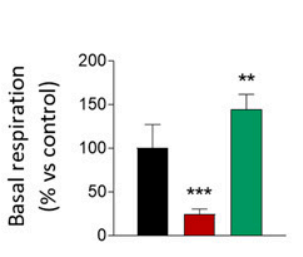
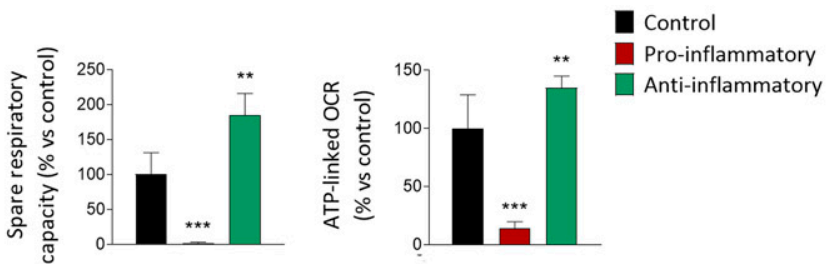

\section{re}
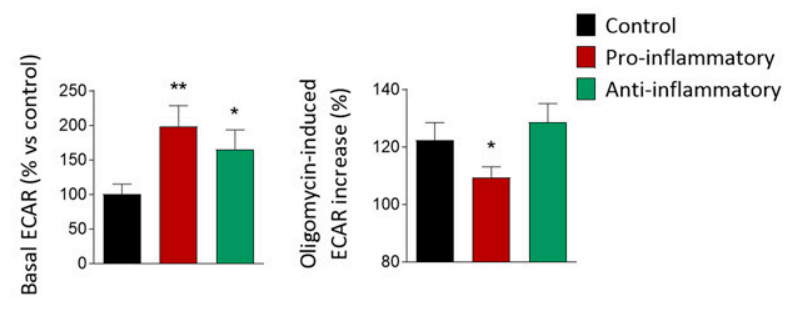

$E$

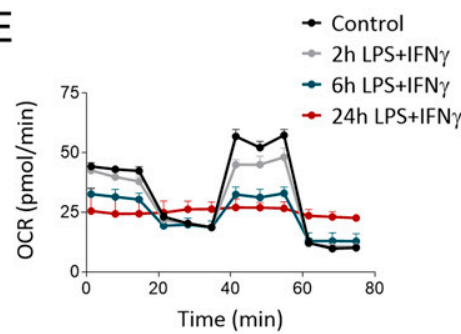

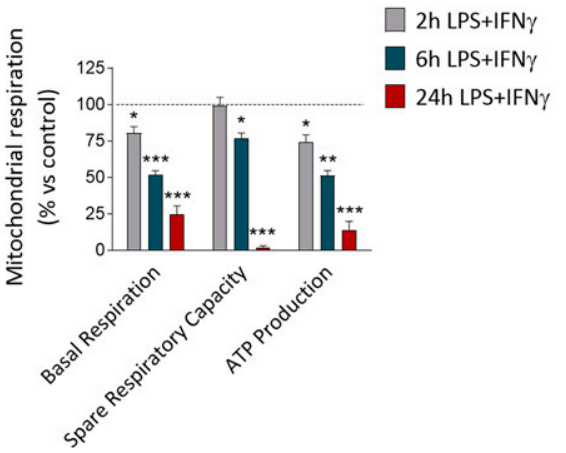

FIGURE 1. Robust metabolic reprogramming to glycolytic pathway after $24 \mathrm{~h}$ of proinflammatory stimulation in microglia.

(A) Representative experiment of OCR measurements in control microglia and in microglia after $24 \mathrm{~h}$ of proinflammatory (LPS plus IFN- $\gamma$ ) and antiinflammatory (IL-4 plus IL-13) stimulations. Histograms show metabolic parameters obtained by the analysis of this metabolic profile compared with control cells ( $n=7$ independent experiments). (B) ECAR measurement obtained by the secretion of lactate of control microglia and microglia after 24-h incubation with pro- and anti-inflammatory factors $(n=7)$. Histograms show the basal level of this parameter as well as the increase in provocation by the acute treatment with oligomycin relative to the control cells. Statistical analysis was performed by one-way ANOVA followed by Bonferroni post hoc test. (C) Ratio between the basal OCR and the basal glycoPER control and both proinflammatory and anti-inflammatory microglia after $24 \mathrm{~h}$ of stimulation. These parameters were obtained using the XF Glycolytic Rate Assay test ( $n=3$ independent experiments). (D) Metabolic profile of microglia after acute treatment with LPS and IFN- $\gamma$. This treatment was added after the basal measurement of OCR and prior to the addition of oligomycin $(n=3)$. (E) Metabolic profile of microglia treated during different time lapses with LPS and IFN- $\gamma$. Histograms show the metabolic parameters compared with control cells $(n=3)$. Data are presented as means \pm SEM. Unless otherwise stated, a Student $t$ test was used to analyze the data. ${ }^{*} p<0.05,{ }^{* *} p<0.005,{ }^{* * *} p<0.001$.

with respiration inhibited by the reverse operation of $\mathrm{F}_{0} \mathrm{~F}_{1}-\mathrm{ATP}$ synthase and the adenine nucleotide translocase (ANT), which pump $\mathrm{H}^{+}$out of the matrix (35). To test this hypothesis, we analyzed the impact of the $\mathrm{F}_{0} \mathrm{~F}_{1}$-ATP synthase and ANT in cell viability and $\Delta \Psi \mathrm{m}$. Indeed, treatment of LPS plus IFN$\gamma$-stimulated microglia with oligomycin, an $\mathrm{F}_{0} \mathrm{~F}_{1}$-ATP synthase inhibitor, and induced a significant increase in microglia cell death (Fig. 2D). In contrast, bongkrekic acid, an inhibitor of ANT (36), did not induce microglia cell death (Fig. 2D). Accordingly, a role of $\mathrm{F}_{0} \mathrm{~F}_{1}$-ATP synthase in mitochondrial $\Delta \Psi \mathrm{m}$ maintenance in proinflammatory microglia was further corroborated by live cell imaging of tetramethylrhodamine ethyl ester, a fluorescent dye that accumulates in active mitochondria. Addition of oligomycin induced a significant decrease in $\Delta \Psi \mathrm{m}$ in proinflammatory microglia but not in control microglia (Fig. 2E), supporting the idea that the former's mitochondrial integrity depends on the activity of the ATP synthase.

\section{Mitochondrial fission inhibition does not reverse mitochondrial metabolic reprogramming}

Previous data have showed that activation of microglia with LPS induced a transient shortening of mitochondria by fission at $2 \mathrm{~h}$. Similarly, we detected a shortening of mitochondrial length at $2 \mathrm{~h}$ after LPS plus IFN- $\gamma$ treatment, an effect that was reverted in the presence of the mitochondrial fission inhibitor 
A
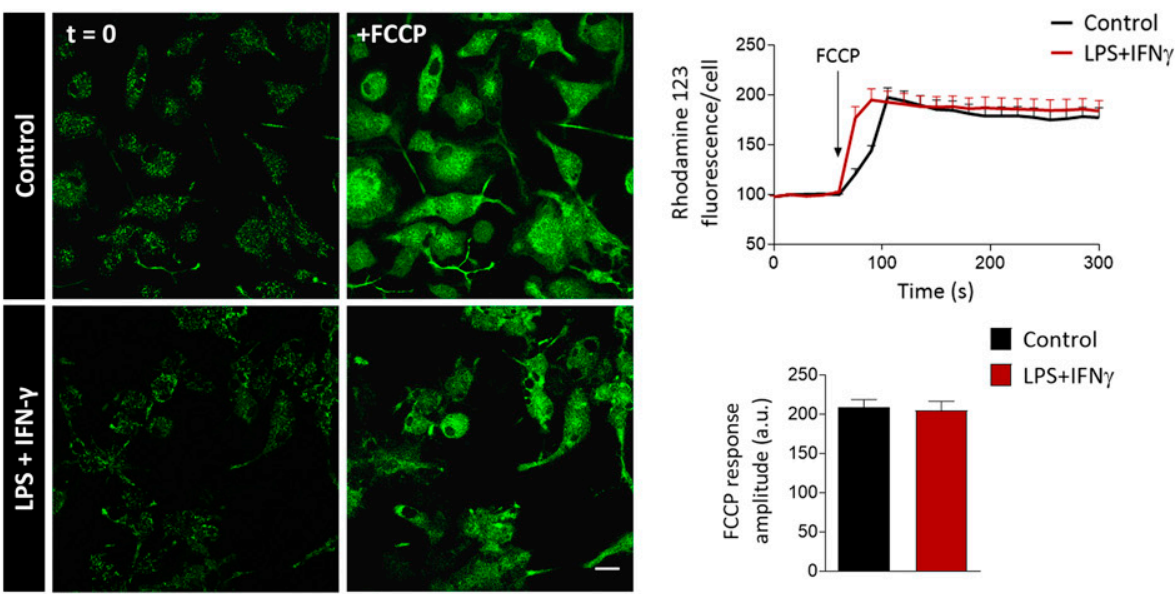

B
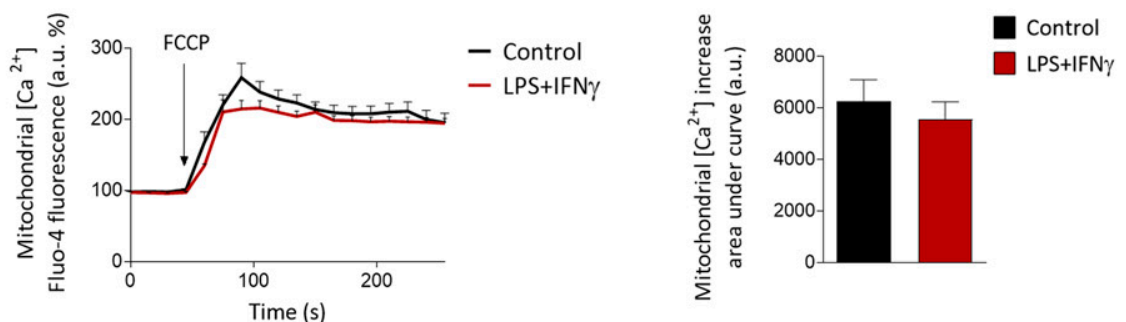

C

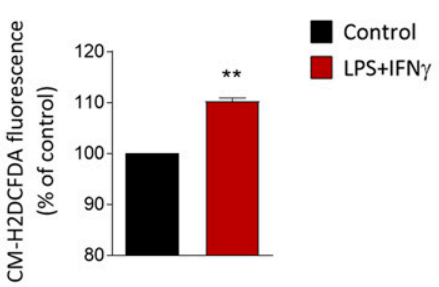

E

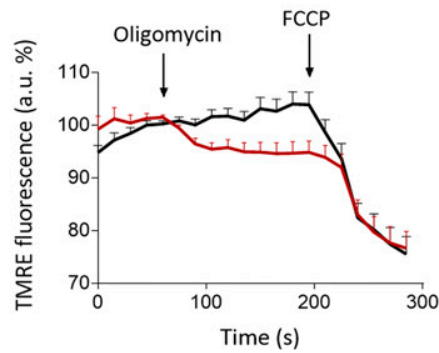

D

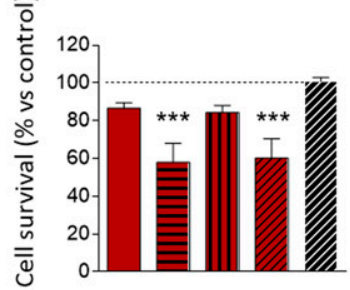

LPS+IFN $\gamma++++-$

Oligomycin - + - + +

Bongkrekic acid - - + + +

FIGURE 2. Proinflammatory microglia maintain mitochondrial integrity upon FOF1-ATP synthase activity blockade.

(A) Measurement of mitochondrial potential represented as the increase in cytoplasmic Rh123 fluorescence measured after acute exposure to FCCP in control and LPS plus IFN- $\gamma$-treated microglia ( $n=50-75$ cells from three independent experiments). Scale bar, $20 \mu M$. (B) Measurement of mitochondrial-specific calcium represented as the increase in cytoplasmic Fluo-4 fluorescence in a $\mathrm{Ca}^{2+}$-free medium after exposure to FCCP in control and LPS plus IFN- $\gamma$-treated cells ( $n=50-75$ cells from three independent experiments). (C) ROS quantification in control and proinflammatory cells ( $n=3$ experiments performed in triplicate). (D) Microglial viability after 24-h treatment with LPS plus IFN- $\gamma$, oligomycin, and/or bongkrekic acid compared with control cells. One-way ANOVA followed by Bonferroni post hoc analysis was used ( $n=3$ experiments performed in triplicate). (E) Effect of FOF1-ATP synthase inhibitor oligomycin in mitochondrial potential of control and LPS plus IFN- $\gamma$-treated cells $(n=70-80$ cells from three independent experiments). Data are presented as means $\pm \mathrm{SEM}$. Unless otherwise stated, a Student $\mathrm{t}$ test was used to analyze the data. ${ }^{* *} p<0.005,{ }^{* \star *} p<0.001$ 
A

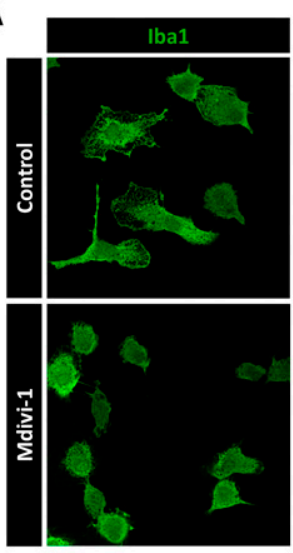

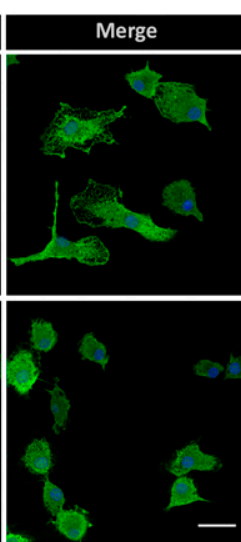

Control

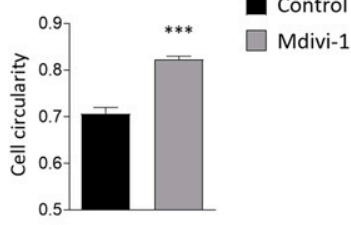

D

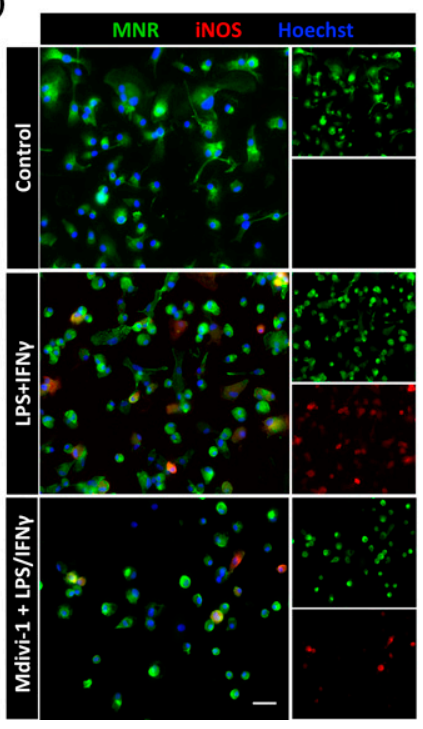

$B$

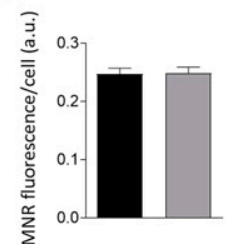

C

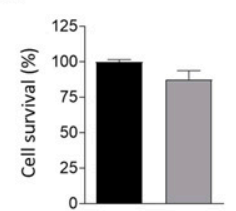

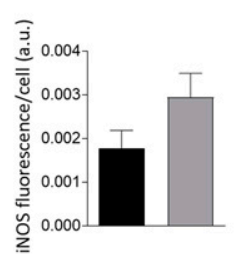

Control

$\square$ Mdivi-1

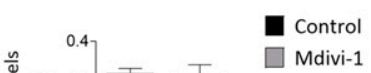

$\mathrm{E}$
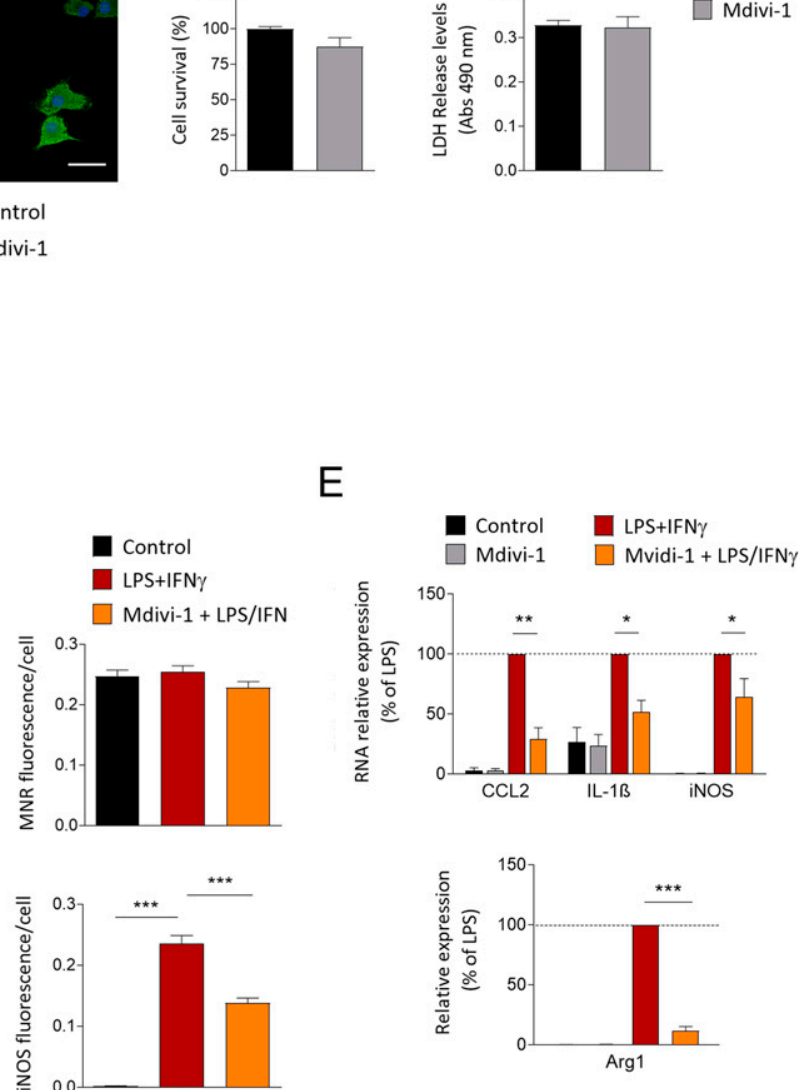

FIGURE 3. Mitochondrial fission inhibition reduces the microglial inflammatory markers.

(A) Representative images of Iba1 ${ }^{+}$control and Mdivi-1-treated microglia. Right column shows Iba1 ${ }^{+}$and DAPI staining (merge). Scale bar, $25 \mu \mathrm{m}$. Histogram represents the circularity of the cells as calculated by ImageJ software ( $n=200$ cells from three independent experiments). (B) Expression of anti-inflammatory (MNR) and proinflammatory mediators (iNOS) in control and Mdivi-1-treated microglia ( $n=4$ independent experiments performed with duplicates). (C) Microglial viability in control and Mdivi-1 cells, measured by both calcein assay and the quantification of lactate dehydrogenase release to the medium, indicative of cell death ( $n=3$ experiments performed in triplicate). (D) Representative immunostaining of MNR and iNOS in control microglia as well as in cells treated with LPS plus IFN- $\gamma$ or Mdivi-1 and LPS plus IFN- $\gamma$. Scale bar, $40 \mu m$. Histograms represent the mean fluorescence of the staining per cell ( $n=$ four independent experiments performed with duplicates). One-way ANOVA followed by Bonferroni post hoc analysis was used. (E) Quantitative real-time PCR of proinflammatory (left) and an anti-inflammatory mediator (right) in control microglia as well as in microglia treated with Mdivi-1, LPS plus IFN- $\gamma$, and Mdivi-1 plus LPS plus IFN- $\gamma(n=4$ experiments performed in duplicate). Data are expressed relative to the expression in LPS plus IFN- $\gamma$. One-way ANOVA followed by Bonferroni post hoc analysis was used. Data are presented as means \pm SEM. Unless otherwise stated, a Student $t$ test was used to analyze the data. ${ }^{\star} p<0.05,{ }^{* \star} p<0.005,{ }^{* \star *} p<0.001$.

Mdivi-1 (9, 37) (Supplemental Fig. 1). Because microglia metabolic switch has been associated previously with mitochondrial fission (9), we further analyzed this hypothesis in our cultures using Mdivi-1. First, we assessed whether the Mdivi-1 treatment would have an effect on microglia itself. A $24-\mathrm{h}$ treatment with Mdivi-1 $(50 \mu \mathrm{M})$ provoked a morphological change in microglia, with a significant increase in cytoplasm circularity, indicating that Mdivi-1 treatment induces an 
TABLE I. List of primers for quantitative real-time PCR

\begin{tabular}{lcc}
\multicolumn{1}{c}{ Target gene } & Forward sequence $\left(5^{\prime}->\mathbf{3}^{\prime}\right)$ & Reverse sequence $\left(5^{\prime}->\mathbf{3}^{\prime}\right)$ \\
\hline Arg1 & GGATTGGCAAGTGATGGAA & CGACATCAAAGCTCAGGTGAA \\
Ccl2 & AGCAGCAGGTGCCCAAA & TTCTTGGGGTAGCACAGAC \\
Il1b & TGGCAACTGTTCCTGACTCA & GGGTCCGTCAACTTCAAAGAAC \\
Nos2 & GAGGAGCAGGTGGAGACTA & GGAAAAGACTGCACCGAAGATA \\
Housekeeping gene & Forward sequence $\left(5^{\prime}->\mathbf{3}^{\prime}\right)$ & Reverse sequence $\left(\mathbf{5}^{\prime}->\mathbf{3}^{\prime}\right)$ \\
Hprt2 & CAGTACAGCCCCAAATGGTTA \\
Ppia & AGGGTTCCTCCTTTCACAGAA & AGTCTGGCCTGTATCCAACA \\
\hline
\end{tabular}

Primers were designed using Primer Express Software (Applied Biosystems) at exon junctions to avoid genomic DNA amplification.

amoeboid morphology (Fig. 3A). Although this characteristic is generally associated with cell activation, we found no differences in the basal expression of proinflammatory (iNOS) or anti-inflammatory markers (MNR) (Fig. 3B) nor in cell viability (Fig. 3C). However, Mdivi-1 reduced significantly the increased expression of iNOS in response to LPS and IFN- $\gamma$ stimulation (Fig. 3D). We further analyzed by qPCR the expression of different proinflammatory mediators. Treating microglia with LPS plus IFN- $\gamma$ induced an increase in all the proinflammatory gene expression assessed (Ccl2, Illb, iNOS) (Table I) with respect to control cells, and also the expression of Argl, an anti-inflammatory mediator that is known to carry an opposite function to iNOS regarding cellular metabolism (14). The treatment with Mdivi-1 significantly reduced proinflammatory mediators overexpression as well as that of Argl (Fig. 3E). These results suggest that Drpl-dependent mitochondrial fission is potentially involved in microglial activation.
Next, we checked whether the impact of Mdivi-1 on microglial activation could affect microglia metabolic reprogramming. In addition to the blockage of Drpl mitochondrial fission, Mdivi-1 provoked an acute and reversible inhibition of mitochondrial complex I in neurons (38). To determine the direct impact of Mdivi-1 on microglial bioenergetics, we treated microglia with Mdivi-1 for $1 \mathrm{~h}$ and $24 \mathrm{~h}$, and we measured microglial OCR. We also observed a reduction in mitochondrial respiration at $1 \mathrm{~h}$; however, the effect was transient and disappeared at $24 \mathrm{~h}$ (Fig. 4A, 4B). Next, we analyzed the impact of Mdivi-1 on the metabolic switch of proinflammatory microglia. Treatment with Mdivi-1 did not increase OCR levels in cells stimulated with LPS and IFN- $\gamma$ to recover the normal bioenergetics profile (Fig. 4B). These results indicate that, although Drpl-mediated mitochondrial fission could modulate or contribute to microglia activation, it does not play an active role in the metabolic switch produced in inflammatory paradigms.
A
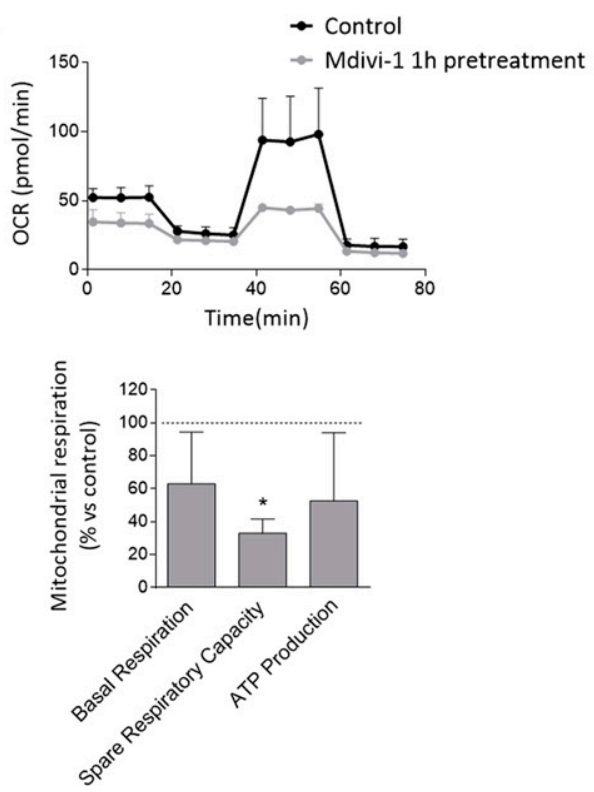

B
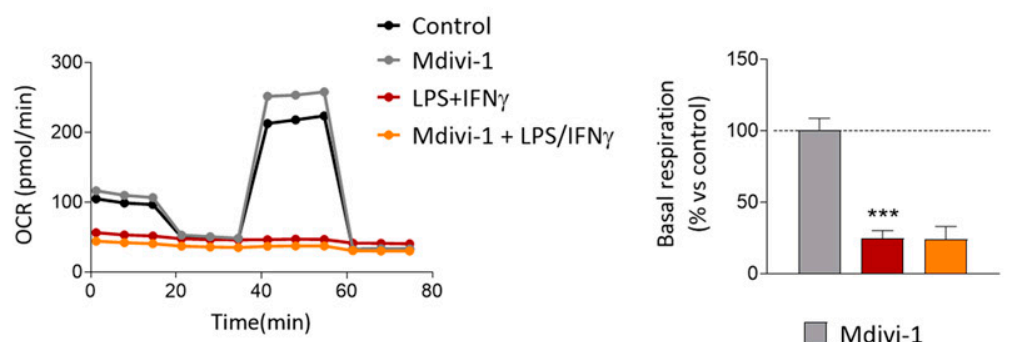

Mdivi-1
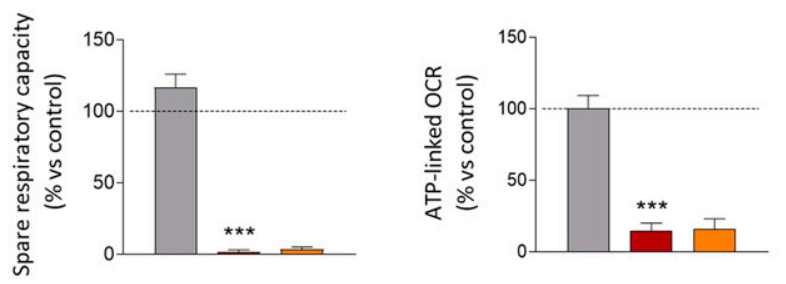

FIGURE 4. Mitochondrial fission inhibition does not reverse mitochondrial metabolic reprogramming.

(A) Metabolic profile of control and microglia treated for $1 \mathrm{~h}$ with Mdivi-1 (above). Histogram shows the metabolic parameters related to OCR of Mdivi-1-treated cells compared with the control ones $(n=3)$. (B) Metabolic profile of control microglia as well as microglia treated for $24 \mathrm{~h}$ with Mdivi-1, LPS plus IFN- $\gamma$, and Mdivi-1 plus LPS plus IFN- $\gamma$. Histograms show the metabolic parameters related to OCR compared with control microglia $(n=3)$. Data are presented as means \pm SEM. One-way ANOVA followed by Bonferroni post hoc analysis was used. ${ }^{*} p<0.05,{ }^{\star \star *} p<0.001$. 
A

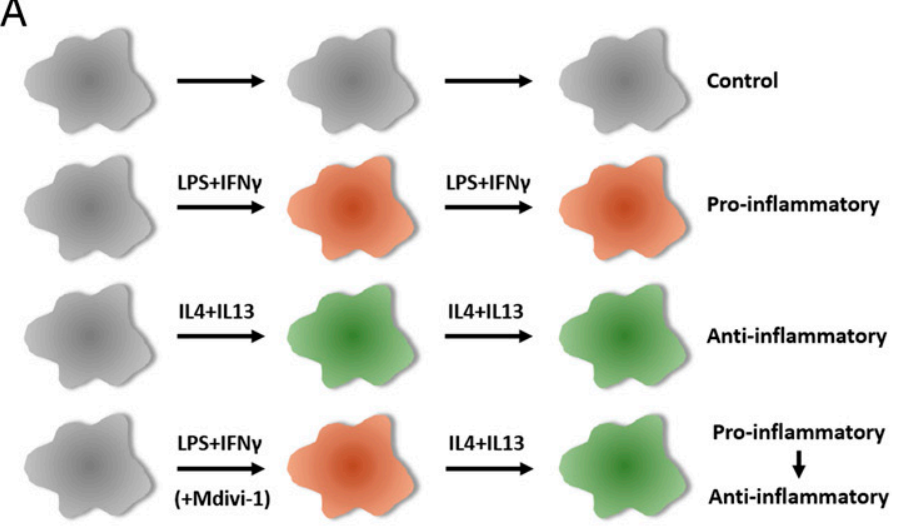

$\mathrm{B}$

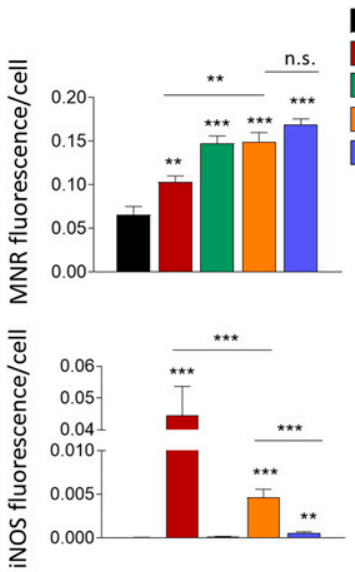

Control

LPS+IFN $\gamma$

IL-4 + IL-13

LPS+IFN $\gamma-->$ ILS

Mdivi-1 + LPS/IFN $\gamma-->$ ILs
C

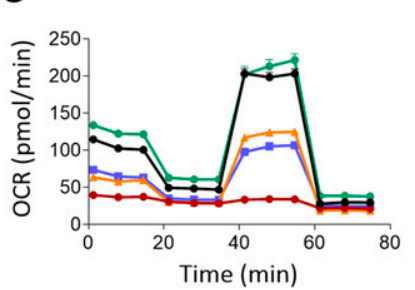

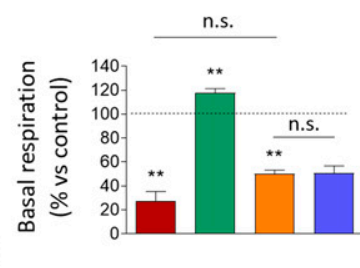
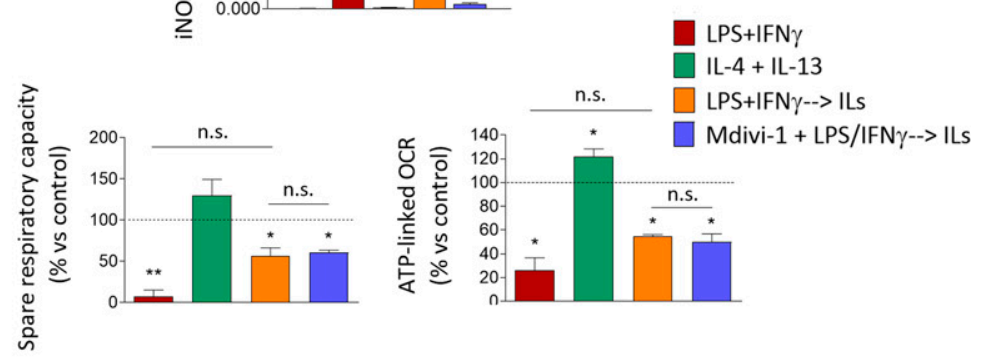

FIGURE 5. Mdivi-1 treatment does not promote microglial repolarization.

(A) Scheme of the conditions used to study the role of Mdivi-1 in the repolarization capacity of microglia. Each treatment indicated lasts $24 \mathrm{~h}$. Antiinflammatory microglia (treated with ILs) were compared with microglia that were primed for $24 \mathrm{~h}$ with LPS plus IFN- $\gamma$ before $24-\mathrm{h}$ treatment with ILs. (B) Expression of anti-inflammatory (MRC1) and proinflammatory (iNOS) markers in every condition of the repolarization experiment. Histograms represent the mean \pm SEM of the marker fluorescence intensity per cell ( $n=3$ experiments performed in triplicate). (C) Metabolic profile (left) of microglia treated as schematized in (A). Histograms (right) show metabolic parameters regarding OCR of the cells $(n=3)$. Data were expressed as fold change versus control nontreated microglia. Data are presented as means \pm SEM. ${ }^{*} p<0.05,{ }^{* \star} p<0.005,{ }^{* \star *} p<0.001$ by Student $t$ test.

\section{Mdivi-1 treatment does not improve microglial repolarization}

Redirection of microglia from a detrimental to a regenerative phenotype is a major concept for developing new therapies targeting these cells (39). NO, the product of iNOS activity, blunts mitochondrial respiration of proinflammatory macrophages, and this dysfunction prevents editing the macrophage toward an anti-inflammatory phenotype. Thus, inhibiting NO production improves mitochondrial metabolic impairment and macrophages reprogramming toward an anti-inflammatory phenotype (40). Given that Mdivi-1 reduced iNOS expression in proinflammatory microglia, we assessed the capacity of Mdivi-1 to promote repolarization in microglia. To that end, microglia cells were primed for $24 \mathrm{~h}$ with LPS plus IFN- $\gamma$ (with or without Mdivi-1, to reduce NO production) and then treated $24 \mathrm{~h}$ with anti-inflammatory cytokines (see scheme in Fig. 5A). We first compared the IL-4 plus IL-13-induced response in cells previously primed with LPS plus IFN- $\gamma$ with the response of cells exposed to antiinflammatory stimulus only. After exposure to IL-4 plus IL-13, microglia primed with LPS plus IFN- $\gamma$ exhibited efficient upregulation in the expression of the anti-inflammatory marker MNR and downregulation in the expression of iNOS (Fig. 5B). The expression of iNOS and MNR were further reduced or increased, respectively, in cells treated with Mdivi-1 during LPS plus IFN- $\gamma$ priming (Fig. 5B). When the metabolic profile of the cells was assessed using the Seahorse XFe96 Analyzer, we observed that microglia primed with LPS plus IFN- $\gamma$ partially recovered the mitochondrial respiration after exposure to IL-4 plus IL-13 (Fig. 5C). However, Mdivi-1 treatment during LPS plus IFN- $\gamma$ priming did not further enhance the final OCR and therefore the OXPHOS activity (Fig. 5C). These results do not support the implication of mitochondrial dynamics in the repolarization process of microglia.

\section{DISCUSSION}

Immunometabolism has recently emerged as an important focus of research, as it opens a novel therapeutic approach for inflammatory and autoimmune diseases. To conduct some responses, effector immune cells, such as microglia/macrophages, undergo a metabolic reprogramming process $(5,7)$. In this study, we have monitored this effect in primary microglia and its consequences in mitochondrial integrity; moreover, we have checked whether this metabolic switch is associated with mitochondrial fission. It is noteworthy to mention that the ablation of OXPHOS in active cells is not due to a challenge to 
mitochondrial integrity. Moreover, we have shown that Drpldependent mitochondrial fission, although potentially involved in microglial activation, does not play an essential role in metabolic reprogramming of microglia.

Upon proinflammatory stimulation, cells are able to redirect their entire metabolic processes to the glycolytic pathway to rapidly obtain energy. This process is an outcome of different molecular pathways; nevertheless, the precise mechanisms involved are yet to be defined. In this study, we have observed that, although the OXPHOS machinery is completely halted, this is not associated with mitochondrial damage or dysfunction nor with microglia cell death. This is opposed to what was observed when the Warburg effect was first described; it was hypothesized that dysfunctional mitochondria would be the reason underlying the switch in metabolism and, eventually, the development of cancer cells (41). However, recent studies have highlighted the importance of mitochondria regarding the production of ROS as agents to support not only the development of cancer cells but also the proinflammatory state of macrophages $(42,43)$, suggesting no mitochondrial dysfunctionality. Thus, TLR activation in macrophages induced mitochondrial ROS generation, an essential step for efficient intracellular bacteria killing (43). We observed that $\Delta \Psi \mathrm{m}$ was maintained in proinflammatory microglia through the reverse operation of $\mathrm{F}_{0} \mathrm{~F}_{1}$-ATP synthase and that this protects microglia from cell death. Indeed, the blockage of complex I, III, and IV abolishes $\mathrm{H}^{+}$translocation and it would lead to a transient drop in $\Delta \Psi \mathrm{m}$. However, the F1 subunit of $\mathrm{F}_{0} \mathrm{~F}_{1}-\mathrm{ATP}$ synthase can hydrolyze mitochondrial ATP under these circumstances and drives the F0 rotor to pump $\mathrm{H}^{+}$out of the matrix to be able to maintain the $\Delta \Psi \mathrm{m}$ (44). Thus, the mitochondria of proinflammatory microglia would become consumers rather than ATP generators, further increasing the energetic demand of these cells (45). We have not found any essential role in the ANT reversal activity, which has also been described as key in the $\Delta \Psi \mathrm{m}$ maintenance process in similar paradigms (35).

Signaling events mediated by extracellular signals can regulate the metabolic pathways in immune cells such as macrophages or microglia (46). Accordingly, diverse cellular functions have been associated with metabolic reprogramming, including those related to mitochondrial function in general. Previous data suggested that mitochondrial dynamics contribute to this mechanism (9). Our results demonstrated that Mdivi-1, a putative mitochondrial fission inhibitor, reduced the enhancement of markers associated with microglial activation after LPS and IFN- $\gamma$ exposure. This effect on microglial activation is in agreement with other studies, even in other paradigms of treatment $(9,37)$. Drpl-mediated mitochondrial fission has been associated with enhanced activation of both p38 and NF-к $\beta$, both mediators of signaling cascades leading to the expression of proinflammatory genes, in a paradigm of diabetic nephropathy (47). Moreover, blocking Drpl-dephosphorylation with oleuropein reduced the production of proinflammatory factors in microglia as well (48). In contrast, blockage of mitochondrial fission with Mdivi-1 did not avoid the microglial metabolism switch to glycolysis upon LPS plus IFN- $\gamma$ exposure, nor did it provoke any effect in the control cells. We concluded that mitochondria fission does not contribute to the metabolic switch in microglia. This result is apparently at odds with previous results (9). The contradiction may be explained on the basis of the different paradigm used; in the Nair and colleagues study (9), microglia was pretreated with Mdivi-1 (25 $\mu \mathrm{M})$ for $1 \mathrm{~h}$ before LPS stimulation (100 ng/ml. $24 \mathrm{~h}$ ). We, in contrast, blocked mitochondrial fission with Mdivi-1 $(50 \mu \mathrm{M})$ during the whole LPS $(10 \mathrm{ng} / \mathrm{ml})$ plus IFN- $\gamma(20 \mathrm{ng} / \mathrm{ml})$ treatment $(24 \mathrm{~h})$.

Distinct arginine metabolism plays a key role in the metabolic plasticity of immune cells. Proinflammatory microglia convert arginine into NO trough iNOS activity, which is increased in this phenotype $(14,49)$. It has been described that the upregulation of iNOS and the resulting generation of NO contributes to the impairment of mitochondrial respiration both in immune cells and astrocytes (50,51). Moreover, editing macrophage and microglia (re)polarization is emerging as a new therapeutic approach, and iNOS has been described as a target. Actually, iNOS inhibition improves metabolic and phenotypic reprograming to anti-inflammatory macrophages (40). Despite that Mdivi-1 treatment consistently reduced iNOS expression in proinflammatory microglia, we did not detect any significant improvement on mitochondrial respiration. There are two possible interpretations. The complete blockage of iNOS activity and total abolishment of NO production, as observed with the iNOS inhibitor 1400W (52), could be required to prevent the metabolic switch. In this sense, Mdivi-1 only partially reduced iNOS expression in proinflammatory microglia. Alternatively, signaling pathways controlling metabolic switch could differ from those regulating phenotypic and inflammatory expression. Indeed, iNOS inhibition does not affect phenotypic polarization of cells nor the inflammatory cytokine secretion of macrophages (40). Accordingly, the effect of Mdivi-1 on proinflammatory gene expression does not produce any change on metabolism. For instance, Mdivi-1 rapidly and reversibly attenuated complex I-dependent reverse electron transfer-mediated ROS production by brain mitochondria oxidizing succinate (20).

In summary, the current study sheds some light into the role of mitochondria in the metabolic reprogramming process in microglia. Proinflammatory stimuli dampen mitochondrial function without compromising their integrity, and our results point to $\mathrm{F}_{0} \mathrm{~F}_{1}$-ATP synthase as a key regulator of mitochondrial potential and cell viability maintenance in these conditions. However, we concluded that mitochondrial dynamics, fusion-fission, although potentially involved in proinflammatory gene expression, do not contribute to microglial glycolytic switch after proinflammatory stimulation.

\section{DISCLOSURES}

The authors have no financial conflicts of interest. 


\section{ACKNOWLEDGMENTS}

We kindly acknowledge the Microscopy Facilities of both the University of the Basque Country and Achucarro Basque Center for Neuroscience for the technical support.

\section{REFERENCES}

1. Davalos, D., J. Grutzendler, G. Yang, J. V. Kim, Y. Zuo, S. Jung, D. R. Littman, M. L. Dustin, and W. B. Gan. 2005. ATP mediates rapid microglial response to local brain injury in vivo. Nat. Neurosci. 8: 752-758.

2. Nimmerjahn, A., F. Kirchhoff, and F. Helmchen. 2005. Resting microglial cells are highly dynamic surveillants of brain parenchyma in vivo. Science 308: 1314-1318.

3. Ransohoff, R. M., and J. El Khoury. 2015. Microglia in health and disease. Cold Spring Harb. Perspect. Biol. 8: a020560.

4. Xue, J., S. V. Schmidt, J. Sander, A. Draffehn, W. Krebs, I. Quester, D. De Nardo, T. D. Gohel, M. Emde, L. Schmidleithner, et al. 2014. Transcriptome-based network analysis reveals a spectrum model of human macrophage activation. Immunity 40: 274-288.

5. Andrejeva, G., and J. C. Rathmell. 2017. Similarities and distinctions of cancer and immune metabolism in inflammation and tumors. Cell Metab. 26: 49-70.

6. Stienstra, R., R. T. Netea-Maier, N. P. Riksen, L. A. B. Joosten, and M. G. Netea. 2017. Specific and complex reprogramming of cellular metabolism in myeloid cells during innate immune responses. Cell Metab. 26: 142-156.

7. Kelly, B., and L. A. J. O'Neill. 2015. Metabolic reprogramming in macrophages and dendritic cells in innate immunity. Cell Res. 25: 771-784.

8. Lauro, C., and C. Limatola. 2020. Metabolic reprograming of microglia in the regulation of the innate inflammatory response. Front. Immunol. 11: 493.

9. Nair, S., K. S. Sobotka, P. Joshi, P. Gressens, B. Fleiss, C. Thornton, C. Mallard, and H. Hagberg. 2019. Lipopolysaccharide-induced alteration of mitochondrial morphology induces a metabolic shift in microglia modulating the inflammatory response in vitro and in vivo. Glia 67: 1047-1061.

10. Hu, Y., W. Mai, L. Chen, K. Cao, B. Zhang, Z. Zhang, Y. Liu, H. Lou, S. Duan, and Z. Gao. 2019. mTOR-mediated metabolic reprogramming shapes distinct microglia functions in response to lipopolysaccharide and ATP. Glia 68: 1031-1045.

11. Holland, R., A. L. McIntosh, O. M. Finucane, V. Mela, A. RubioAraiz, G. Timmons, S. A. McCarthy, Y. K. Gun'ko, and M. A. Lynch. 2018. Inflammatory microglia are glycolytic and iron retentive and typify the microglia in APP/PS1 mice. Brain Behav. Immun. 68: 183-196.

12. Van den Bossche, J., L. A. O'Neill, and D. Menon. 2017. Macrophage immunometabolism: Where are we (going)? Trends Immunol. 38: 395-406.

13. Bernier, L. P., E. M. York, and B. A. MacVicar. 2020. Immunometabolism in the brain: how metabolism shapes microglial function. Trends Neurosci. 43: 854-869.

14. Rath, M., I. Müller, P. Kropf, E. I. Closs, and M. Munder. 2014. Metabolism via arginase or nitric oxide synthase: two competing arginine pathways in macrophages. Front. Immunol. 5: 532.

15. Westermann, B. 2010. Mitochondrial fusion and fission in cell life and death. Nat. Rev. Mol. Cell Biol. 11: 872-884.

16. Ishihara, N., M. Nomura, A. Jofuku, H. Kato, S. O. Suzuki, K. Masuda, H. Otera, Y. Nakanishi, I. Nonaka, Y. Goto, et al. 2009. Mitochondrial fission factor Drpl is essential for embryonic development and synapse formation in mice. Nat. Cell Biol. 11: 958-966.
17. Knott, A. B., G. Perkins, R. Schwarzenbacher, and E. Bossy-Wetzel. 2008. Mitochondrial fragmentation in neurodegeneration. Nat. Rev. Neurosci. 9: 505-518.

18. Cho, D. H., T. Nakamura, and S. A. Lipton. 2010. Mitochondrial dynamics in cell death and neurodegeneration. Cell. Mol. Life Sci. 67: 3435-3447.

19. Reddy, P. H., T. P. Reddy, M. Manczak, M. J. Calkins, U. Shirendeb, and P. Mao. 2011. Dynamin-related protein 1 and mitochondrial fragmentation in neurodegenerative diseases. Brain Res. Brain Res. Rev. 67: 103-118.

20. Smirnova, E., L. Griparic, D. L. Shurland, and A. M. van der Bliek. 2001. Dynamin-related protein Drp1 is required for mitochondrial division in mammalian cells. Mol. Biol. Cell 12: 2245-2256.

21. Fonseca, T. B., Á. Sánchez-Guerrero, I. Milosevic, and N. Raimundo. 2019. Mitochondrial fission requires DRP1 but not dynamins. Nature 570: E34-E42.

22. Cassidy-Stone, A., J. E. Chipuk, E. Ingerman, C. Song, C. Yoo, T. Kuwana, M. J. Kurth, J. T. Shaw, J. E. Hinshaw, D. R. Green, and J. Nunnari. 2008. Chemical inhibition of the mitochondrial division dynamin reveals its role in Bax/Bak-dependent mitochondrial outer membrane permeabilization. Dev. Cell 14: 193-204.

23. Domercq, M., M. V. Sánchez-Gómez, C. Sherwin, E. Etxebarria, R. Fern, and C. Matute. 2007. System xc- and glutamate transporter inhibition mediates microglial toxicity to oligodendrocytes. $J$. Immunol. 178: 6549-6556.

24. Bezzi, P., M. Domercq, L. Brambilla, R. Galli, D. Schols, E. De Clercq, A. Vescovi, G. Bagetta, G. Kollias, J. Meldolesi, and A. Volterra. 2001. CXCR4-activated astrocyte glutamate release via TNFalpha: amplification by microglia triggers neurotoxicity. Nat. Neurosci. 4: 702-710.

25. Domercq, M., M. V. Sánchez-Gómez, P. Areso, and C. Matute. 1999. Expression of glutamate transporters in rat optic nerve oligodendrocytes. Eur. J. Neurosci. 11: 2226-2236.

26. Domercq, M., B. Szczupak, J. Gejo, V. Gómez-Vallejo, D. Padro, K. B. Gona, F. Dollé, M. Higuchi, C. Matute, J. Llop, and A. Martín. 2016. PET imaging with [(18)F]FSPG evidences the role of system $\mathrm{xc}(-)$ on brain inflammation following cerebral ischemia in rats. Theranostics 6: 1753-1767.

27. Vandesompele, J., K. De Preter, F. Pattyn, B. Poppe, N. Van Roy, A. De Paepe, and F. Speleman. 2002. Accurate normalization of realtime quantitative RT-PCR data by geometric averaging of multiple internal control genes. Genome Biol. 3: RESEARCH0034.

28. Corona, J. C., and M. R. Duchen. 2014. Mitochondrial bioenergetics assessed by functional fluorescence dyes. Neuromethods 90: 161-176.

29. Hardingham, G. E., Y. Fukunaga, and H. Bading. 2002. Extrasynaptic NMDARs oppose synaptic NMDARs by triggering CREB shutoff and cell death pathways. Nat. Neurosci. 5: 405-414.

30. Yan, J., C. P. Bengtson, B. Buchthal, A. M. Hagenston, and H. Bading. 2020. Coupling of NMDA receptors and TRPM4 guides discovery of unconventional neuroprotectants. Science 370: eaay3302.

31. Frey, T. G., and C. A. Mannella. 2000. The internal structure of mitochondria. Trends Biochem. Sci. 25: 319-324.

32. Orihuela, R., C. A. McPherson, and G. J. Harry. 2015. Microglial M1/ M2 polarization and metabolic states. Br. J. Pharmacol. 173: 649-665.

33. Everts, B., E. Amiel, S. C. C. Huang, A. M. Smith, C. H. Chang, W. Y. Lam, V. Redmann, T. C. Freitas, J. Blagih, G. J. W. van der Windt, et al. 2014. TLR-driven early glycolytic reprogramming via the kinases TBK1-IKKe supports the anabolic demands of dendritic cell activation. Nat. Immunol. 15: 323-332.

34. Brocard, J. B., M. Tassetto, and I. J. Reynolds. 2001. Quantitative evaluation of mitochondrial calcium content in rat cortical neurones following a glutamate stimulus. J. Physiol. 531: 793-805.

35. Chinopoulos, C. 2011. Mitochondrial consumption of cytosolic ATP: not so fast. FEBS Lett. 585: 1255-1259. 
36. Henderson, P. J., and H. A. Lardy. 1970. Bongkrekic acid. An inhibitor of the adenine nucleotide translocase of mitochondria. J. Biol. Chem. 245: 1319-1326.

37. Katoh, M., B. Wu, H. B. Nguyen, T. Q. Thai, R. Yamasaki, H. Lu, A. M. Rietsch, M. M. Zorlu, Y. Shinozaki, Y. Saitoh, et al. 2017. Polymorphic regulation of mitochondrial fission and fusion modifies phenotypes of microglia in neuroinflammation. Sci. Rep. 7: 4942.

38. Bordt, E. A., P. Clerc, B. A. Roelofs, A. J. Saladino, L. Tretter, V. AdamVizi, E. Cherok, A. Khalil, N. Yadava, S. X. Ge, et al. 2017. The putative Drpl inhibitor mdivi-1 is a reversible mitochondrial complex I inhibitor that modulates reactive oxygen species. Dev. Cell 40: 583-594.e6.

39. Fumagalli, M., M. Lombardi, P. Gressens, and C. Verderio. 2018. How to reprogram microglia toward beneficial functions. Glia 66: 2531-2549.

40. Van den Bossche, J., J. Baardman, N. A. Otto, S. van der Velden, A. E. Neele, S. M. van den Berg, R. Luque-Martin, H. J. Chen, M. C. S. Boshuizen, M. Ahmed, et al. 2016. Mitochondrial dysfunction prevents repolarization of inflammatory macrophages. Cell Rep. 17: 684-696.

41. Warburg, O. 1956. On the origin of cancer cells. Science 123: 309-314.

42. Porporato, P. E., N. Filigheddu, J. M. B. Pedro, G. Kroemer, and L. Galluzzi. 2017. Mitochondrial metabolism and cancer. Cell Res. 28: 265-280.

43. West, A. P., I. E. Brodsky, C. Rahner, D. K. Woo, H. ErdjumentBromage, P. Tempst, M. C. Walsh, Y. Choi, G. S. Shadel, and S. Ghosh. 2011. TLR signalling augments macrophage bactericidal activity through mitochondrial ROS. Nature 472: 476-480.

44. Kinosita, K., Jr., K. Adachi, and H. Itoh. 2004. Rotation of F1ATPase: how an ATP-driven molecular machine may work. Annu. Rev. Biophys. Biomol. Struct. 33: 245-268.
45. Solaini, G., A. Baracca, G. Lenaz, and G. Sgarbi. 2010. Hypoxia and mitochondrial oxidative metabolism. Biochim. Biophys. Acta 1797: 1171-1177.

46. Wang, S., R. Liu, Q. Yu, L. Dong, Y. Bi, and G. Liu. 2019. Metabolic reprogramming of macrophages during infections and cancer. Cancer Lett. 452: 14-22.

47. Zhang, L., L. Ji, X. Tang, X. Chen, Z. Li, X. Mi, and L. Yang. 2015. Inhibition to DRP1 translocation can mitigate p38 MAPK-signaling pathway activation in GMC induced by hyperglycemia. Ren. Fail. 37: 903-910.

48. Park, J., J. S. Min, U. Chae, J. Y. Lee, K. S. Song, H. S. Lee, H. J. Lee, S. R. Lee, and D. S. Lee. 2017. Anti-inflammatory effect of oleuropein on microglia through regulation of Drpl-dependent mitochondrial fission. J. Neuroimmunol. 306: 46-52.

49. Yang, Z., and X. F. Ming. 2014. Functions of arginase isoforms in macrophage inflammatory responses: impact on cardiovascular diseases and metabolic disorders. Front. Immunol. 5: 533.

50. Everts, B., E. Amiel, G. J. W. van der Windt, T. C. Freitas, R. Chott, K. E. Yarasheski, E. L. Pearce, and E. J. Pearce. 2012. Commitment to glycolysis sustains survival of NO-producing inflammatory dendritic cells. Blood 120: 1422-1431.

51. Bolaños, J. P., S. Peuchen, S. J. R. Heales, J. M. Land, and J. B. Clark. 1994. Nitric oxide-mediated inhibition of the mitochondrial respiratory chain in cultured astrocytes. J. Neurochem. 63: 910-916.

52. Garvey, E. P., J. A. Oplinger, E. S. Furfine, R. J. Kiff, F. Laszlo, B. J. R. Whittle, and R. G. Knowles. 1997. 1400W is a slow, tight binding, and highly selective inhibitor of inducible nitric-oxide synthase in vitro and in vivo. J. Biol. Chem. 272: 4959-4963. 